\title{
CIUDADANÍA Y DEMOCRACIA EN LA SOCIEDAD ACTUAL GLOBALIZADA Y COMUNICADA
}

\author{
J. A. L. HERRERÍAS
Catedrático Teoría de la Educación, Facultad de Educación, Universidad Complutense Madrid, UCM
jherrer@edu.ucm.es \\ Artículo presentado en julio/2016 y aceptado en julio/2016 \\ DOI: $10.15628 /$ holos.2016.4728
}

\section{RESUMEN}

Vivimos una cultura mundializada en dos ámbitos complementarios, cuales son el que se refiere a la generalizada experiencia de la tecnología de la comunicación y el de la interdependencia económica de todas las sociedades. Esa experiencia cultural dominante genera un relevante reto a la experiencia ineludible de la convivencia y al proyecto de cómo y para qué educarnos. En este contexto globalizado de racionalidad tecnológica y de funcionalidad económica aparece el riesgo de quedar las personas reducidas a las exigencias que imponen ese guión cultural. Por ello, hay que replantear qué sujeto y qué educación, valiosos, hemos de concebir para dar una respuesta humanista y ética, a dichos retos. La respuesta que aquí se elabora está apoyada en los siguientes aspectos: i) potenciar una educación, apoyada en la dimensión ética de la persona, como autor y actor inalienable de la propia existencia; ii) concretar esa dimensión ética en el esfuerzo por vivir como ciudadano comprometido y responsable, vertiente personal del ser sujeto, y por participar de la democracia, vertiente social del mismo.

PALABRAS CLAVE: cultura, ética, sujeto, ciudadanía, democracia.

\section{CITIZENSHIP AND DEMOCRACY IN TODAY'S SOCIETY GLOBAL AND REPORTED}

\begin{abstract}
We live in a globalized culture in two complementary areas, which are referred to the widespread experience of communication technology and the economic interdependence of all societies. That dominant cultural experience generates a significant challenge to the inescapable experience of coexistence and the project of how and why education. In this globalized context of technological rationality and economic functionality appears the risk of being reduced to the demands imposed by this cultural. Therefore, we must rethink
\end{abstract}

what subject and what education, valuable, we ask to give a humanist and ethical response to these challenges. The answer here offered is based on the following aspects: i) strengthen education, based on the ethical dimension of the person, as author and actor inalienable existence; ii) specify the ethical dimension in the effort to live as a committed and responsible citizen, personal side of being subject, and participate in democracy, social aspect of it.

KEYWORDS: culture, ethics, person, citizenship, democracy. 


\section{PLANTEAMIENTO DEL PROBLEMA}

Nos preocupan los proyectos educativos y las formas de llevarlos a cabo. Ambos ámbitos complementarios, la propuesta y la acción, han de estar ligados, de un lado, a la realidad compartida, y de otro, a las siempre abiertas posibilidades del ser humano. Se trata de combinar contexto y texto, ambiente y persona. En resumen, ¿qué educación para el mundo actual?

Desde esta secuencia discursiva la pregunta ineludible es ¿qué caracteriza nuestro contexto socio cultural? Ese nuestro tiene cierta orientación universal. No tanto porque piense que en todos los kilómetros cuadrados del planeta se vivan las mismas conciencias y experiencias. Sin embargo, sí podemos estar de acuerdo en que en el conjunto de las sociedades hay ciertos aspectos que atraviesan esa variedad y complejidad de experiencias humanas.

Me refiero a lo siguiente. Que nuestro mundo está marcado por la presencia influyente y dominante de la globalización (Sampedro, J. L., 2009 ) y de la comunicación (Castells, 2006). La globalización hace referencia a la real y activa presencia de los proyectos y afanes económicos a lo largo y ancho del mundo. Todas las decisiones y acciones relacionadas con la producción y distribución de bienes inciden de alguna manera en prácticamente todos los rincones del Planeta. El crecimiento económico de un país, o lo contrario, tiene repercusión en otros muchos, en la dirección ya positiva o negativa, que pueda tener la experiencia inicial de que se trate. Sobre todo de aquellos países de mayor producto interior bruto, de mayor riqueza, USA, China, Rusia,... Ios emergentes.

A su vez, el mundo está comunicado, dado que en relación con lo anterior de la incidencia económica, el gran ámbito de investigación, desarrollo, producción y consumo en el mundo entero está relacionado con los medios tecnológicos de la comunicación. Podemos concluir que hay tres factores que en cierta medida uniformizan en la experiencia humana la enorme diferencia de lenguas, tradiciones, historias. El mundo, entonces, se manifiesta concretado en tres construcciones culturales: i) comunicación y mercado, concretados en el dominio pleno del ii) valor económico, como gran estructura de iii) poder que organiza y dinamiza el mundo.

A partir de este análisis y diagnóstico surge una pregunta: ¿Qué humano hay que educar para que sea una persona capaz de realizarse como sujeto, y no quede dominado por la fuerza dominadora de los medios poderosos establecidos?

\section{¿QUÉ PERSONA Y QUÉ EDUCACIÓN PARA RESOONDER A LA REALIDAD ANALIZADA?}

Respeto de la primera y originaria pregunta, ¿qué persona?, la respuesta razonada y demandada en el estado actual de la reflexión crítica es que sea una persona capaz de comprometerse en ser sujeto de su existencia, autor y actor de la experiencia vital propia. Así lo plantean, entre otros investigadores, P. Freire, concientización, (2009), Z. Baumann, cultura líquida, (2013), A. Touraine, sujeto, (2002), en cuyos análisis y reflexiones sobre nuestra cultura subrayan la exigencia de potenciar la conciencia personal como logro capaz de responder adecuadamente a los retos y problemas de nuestro tiempo espiritual cargado de fuerte impronta de potencial dominio y control, manipulación, hacia los seres humanos.

En segundo lugar, si consistimos en ser los realizadores de nuestro proyecto existencial, esforzarnos por ser éticos, ¿qué educación puede prepararnos para ello? Educarse como 
ciudadano y demócrata. Uno se educa ciudadano y demócrata si potencia una conciencia comprometida, respetuosa y autónoma. Esto es, si se realiza como sujeto, capaz de potenciar las tres complementarias racionalidades que proyectan la tensión espiritual del ser humano, la racionalidad cognitiva o epistémica, la racionalidad estética y la racionalidad ética.

Esto es, si consigue educarse una persona no tanto para insertarse cual eslabón en la cadena de la cultura economicista, globalizada y comunicada, que hemos analizado, para ser un elemento útil en la secuencia del mundo programado por quienes pueden, sino proyectarse como un ser capaz de otra expectativa de realidad cultural y más libre y digna experiencia personal.

Personas capaces de ser sujeto de su existencia, animadores de socio culturas más justas, dignas y fraternas; en consecuencia, menos generadoras de injusticia, violencia y hambre. En definitiva, se trata de tomar conciencia del humano, que éticamente hemos de proponernos ser, más allá de saberse miembro de una dominante racionalidad tecnológica de limitado valor humano. Esa racionalidad tecnológica en la que hoy es fácil quedar atrapados, bajo la fuerza de la potencia asumida por la absolutizada variable independiente de nuestra cultura, cual es lo económico, lleva consigo el olvido manipulativo y muy presente de los valores propuesto como horizonte de la realización humana: libertad, igualdad, dignidad y fraternidad. Esa presente y dominante racionalidad tecnológica configura al humano como un existente, activado para la producción, especializado en consumir, que asume vivir explotado para atender los impuestos mecánicos anteriores, y que se define y reconoce como un eslabón imprescindible de la estructural y organizada funcionalidad financiera. En definitiva, un humano, que bajo una pretensión percibida de libertad, igualdad y dignidad, viviría una experiencia de manipulación y control engañosa y mediocre.

\section{LA PERSONA, SUJETO ESPIRITUAL, AUTOR Y ACTOR DE LA EXISTENCIA.}

Ya se ha señalado que estar en el mundo comunicados y globalizados en las experiencias dominantes de la mensajería apantallada, icónica, y de la racionalidad tecnológica del amor estimativo, valor, a la funcionalidad económica, arrastra consigo el riesgo de reducir y fanatizar en el ser humano una anémica morada de la potencial espiritualidad personal. Que corremos el serio riesgo de empobrecer nuestros horizontes de posibilidad de modo que quedemos limitados a las funciones elementales de las exigencias físicas y biológicas de la existencia, seguridad, control, banalización superficial y mediocre de nuestras expresiones y horizontes psico-culturales.

Heidegger en su Carta sobre el humanismo, (2002), recoge una frase del filósofo griego de Éfeso, Heráclito, que además de ser una bella expresión, muestra hondura reflexiva en relación con lo que se trata aquí. Dice: la morada para el hombre es el espíritu, ethos anthropo daimon. Esto es, que allí donde los humanos estamos como en casa, en nuestra morada, en nuestra conciencia, en nuestro bienestar, es en nuestro espíritu, es en el aire que respiramos, que alimenta, nuestro vivir. Entonces, la pregunta es ¿hacia dónde pesa hoy nuestro espíritu, en qué aire respirado hoy nos encontramos a gusto, en nuestra casa? ¿Nos encontramos a nosotros mismos sometidos, doblegados, a las exigencias estructurales de un aire que nos mantiene ajenos a nosotros mismos, absortos por la mensajería y el afán economicista de vivir cual máquinas y dinero transeúnte? En la medida en que lo vivido sea así corremos el riesgo de ser mínimamente sujetos de nuestra existencia, ni autores, ni actores, reales de nuestro estar en el mundo. Un anulado proyecto ético, una vida alienada, sometida por tantos mensajes y tantas necesidades provocadas e interiorizadas. 
La inoculación de una morada espiritual, cargada y lastrada de raquitismo, banalidad y fanatismo, que desde luego no aportan un proyecto de superación y mejora humana.

La alternativa a este contexto avasallador, comunicado y globalizado, exige que nos esforcemos en educarnos y en reconocernos como sujeto comprometido y responsable de nuestra existencia.

\section{RETO EDUCATIVO COMO SUJETO: RACIONALIDAD ÉTICA.}

Una de las grandes enseñanzas de la cultura griega clásica es la referida a lo ético. Que la dimensión más relevante de la existencia tiene que ver con la conciencia de sabernos realizadores de nuestra persona, de nuestro espíritu, de nuestra realidad. La lengua griega utiliza dos términos, en todo iguales, excepto que en un caso se escribe con e breve, épsilon, $\dot{\varepsilon} \Theta o \varsigma$, y en otro con e larga, eta, $\eta \Theta o s$. Curiosamente es la única vocal que tiene dos usos y denominaciones. No ocurre ni con la alfa, a, ni con la iota, i, ni con la omicron, o, ni con la upsilon, u. Y, ¿por qué esa dualidad con la e? Porque necesitaban significar algo en lo que habían caído en la cuenta, referido a la experiencia y conducta humanas, y que manifiesta una profunda y atenta percepción del ser y el hacer del humano.

Sencillamente, los griegos vieron, idear tiene etimológicamente una muy sugerente cercanía con ver, que las acciones del día a día de las personas, ethos, con e breve, cada acto, sumadas en la experiencia generan, en quien realiza esos actos, una segunda naturaleza, una forma de ser, una morada espiritual, un carácter, entonces, la misma palabra con e larga. Así llevan a cabo la expresión lingüística de esta intuición: que la suma de e breves, e + e + e + e,... épsilon + épsilon,... resulta una e larga, una eta: $\dot{\varepsilon}+\dot{\varepsilon}+\dot{\varepsilon}=\eta$. Esta expresión de la lengua griega nos está diciendo que ética significa que consistimos en hacernos, que lo ético de la existencia consiste en tomar conciencia y aceptar que, la persona que vamos siendo a lo largo de nuestro ser y actuar, depende de los actos que vamos decidiendo hacer y ser en el día a día de la vida. Ser ético es tomar conciencia del compromiso y la responsabilidad que nos ha de suponer sabernos realizadores de nuestra propia y personal realidad.

En definitiva, la consecuencia que se deriva de percibirnos como seres éticos se concreta en asumir que estamos exigidos de ser sujetos de nuestra existencia. El más negativo diagnóstico de anemia ética que podemos vivir es el de dejarnos hacer y perder el compromiso y la responsabilidad de hacernos los sujetos de nuestra existencia. Los tiempos de nuestra sociocultura, comunicados y globalizados, que hemos comentado, son un dominante contexto, que con cierta facilidad nos puede provocar una pandemia de raquitismo espiritual colectivo.

¿En qué concretar brevemente acciones válidas para aprender y mantener el esfuerzo de ser sujeto, en cuanto que consciente realizador del proyecto y de las acciones de la propia historia personal? Básicamente, dos tareas complementarias son las que realizadas nos mantienen y fortalecen en el proyecto ético de actuar como sujetos. Ser autor de nuestro propio guión existencial, y desde él, ser actor de ese guión articulado desde uno mismo.

Si somos y vivimos desde nuestra conciencia, y esta es un museo de palabras, una selección crítica y creativa de palabras, que orientan y concretan nuestra acción en el mundo, es desde ahí, ámbito espiritual permanentemente amenazado de asedio y conquista por quienes tienen interés permanente en ello, desde donde hemos de llevar a cabo la autoría de nuestro guión. En eso 
consiste el esfuerzo y el proyecto ético de nuestro estar en el mundo. Que nadie nos escriba el guión de lo que hemos de pensar, hacer, elegir, decidir,... No existimos para ser clientes de circuitos de poder, hoy comunicados y globalizados, funcionalidad económica, y coyunturalmente política, sino para ser libres y dignos esforzados de nuestra realización. Sujetos.

A su vez, la proyección ética de la construcción inalienable de cada uno no se agota en ese inicial ser autor del personal guión existencial, qué quiero, qué deseo, qué elijo, qué decido,... en los diferentes ámbitos de la vida, sino que esa autoría ha de ser completada con las acciones, actor, que llevan a cabo la plasmación de lo elaborado en el guión. Educarnos para ser sujeto, autor y actor de la propia e inalienable presencia en el mundo.

\section{CIUDADANÍA Y DEMOCRACIA, CONCRETAS ACCIONES DE LA RACIONALIDAD ÉTICA.}

Desde la inquietud investigadora y aplicativa de la educación social para responder adecuadamente a los retos actuales señalados lo proyectado pedagógicamente anima una propuesta de compromiso y responsabilidad para llevar a cabo una convivencia valiosa como ciudadano demócrata. Quiere ello decir que hoy no vale con plantearse una educación centrada en el individuo, como potencial agente de servicios a la comunidad, y como ser que necesita potenciar las capacidades abiertas y creativas de la complejidad humana, dimensiones deportivas, estéticas, afectivas, cognitivas, éticas, y los diferentes ámbitos de aplicación de las mismas, en las diferentes profesiones y servicios en que se articula la vida cotidiana de las sociedades.

Además de eso y sobre eso, nuestro complejo mundo comunicado y globalizado, inundado de mensajería y de exigencias y retos económicos interconectados, requiere que nos eduquemos de modo holístico y ético para llegar a ser competentes como ciudadanos implicados de forma comprometida y responsable en las múltiples vertientes en que las hoy nos vemos apelados.

En un pasado, aún muy presente, nos hemos educado para, como sujetos de mente parcelada cognitivamente, saber las respuestas adecuadas para sabernos y reconocernos inmersos en la vida social. Hoy, la señalada complejidad de la vida social y cultural de la sociedad de la comunicación y de la globalización tecnológica y económica requiere que nos eduquemos desde el subrayado de la vertiente ética del ser humano. Eso se concreta en aprender estética, intelectual, afectiva, y éticamente, sobre todo esto último, que estamos exigidos para potenciar nuestra implicación en las experiencias que como ciudadanos, seres de convivencia ineludible, hemos de compartir. Todo este renovado programa requiere concretar el cómo hacer para llevarlo a cabo, asuntos claves que se pueden desgranar paso a paso posteriormente. Sin embargo, la primera e inicial tarea, como en cualquier iniciativa humana de cambio y renovación, consiste en darse cuenta, en tomar conciencia, intensa y profundamente, de que los retos son otros, que los dominantes en el pasado, y que el sujeto que hemos de poner en las tablas del escenario de la convivencia mundial, que deseamos más justa, libre, igual, digna y fraterna, se eduque para esa racionalidad ética comprometida y responsable, brevemente iniciada en estas páginas. Gracias.

\section{REFERENCIAS BIBLIOGRAFICAS.}

1. BAUMANN, Z. La cultura en el mundo de la modernidad líquida. México, F.C.E., 2013. 
2. CASTELLS, M. En qué mundo vivimos: conversaciones con Manuel Castells. Madrid, Alianza Editorial, 2006.

3. FREIRE, P. La educación como práctica de la libertad. Madrid, Siglo XXI, 2009.

4. HEIDEgGER, M. Carta sobre el humanismo. Madrid, Alianza Editorial, 2002.

5. SAMPEDRO, J. L. Economía humanista: algo más que cifras. Barcelona, Debate, 2009.

6. TOURAINE, A. A la búsqueda de sí mismo. Diálogos sobre el sujeto. Barcelona, Paidós, 2002. 